\title{
Dynamical evolution of a scalar field coupling to Einstein's tensor in the Reissner-Nordström black hole spacetime
}

\author{
Songbai Chen*, Jiliang Jing ${ }^{\dagger}$ \\ Institute of Physics and Department of Physics, Hunan Normal University, \\ Changsha, Hunan 410081, People's Republic of China \\ Key Laboratory of Low Dimensional Quantum Structures \\ and Quantum Control of Ministry of Education, Hunan Normal University, \\ Changsha, Hunan 410081, People's Republic of China
}

\begin{abstract}
We study the dynamical evolution of a scalar field coupling to Einstein's tensor in the background of Reissner-Nordström black hole. Our results show that the the coupling constant $\eta$ imprints in the wave dynamics of a scalar perturbation. In the weak coupling, we find that with the increase of the coupling constant $\eta$ the real parts of the fundamental quasinormal frequencies decrease and the absolute values of imaginary parts increase for fixed charge $q$ and multipole number $l$. In the strong coupling, we find that for $l \neq 0$ the instability occurs when $\eta$ is larger than a certain threshold value $\eta_{c}$ which deceases with the multipole number $l$ and charge $q$. However, for the lowest $l=0$, we find that there does not exist such a threshold value and the scalar field always decays for arbitrary coupling constant.
\end{abstract}

PACS numbers: 04.70.Dy, 95.30.Sf, 97.60.Lf

* csb3752@163.com

† jljing@hunnu.edu.cn 


\section{INTRODUCTION}

The dynamical evolution of an external field perturbation around a black hole has been an object of great interest for physicists for the last few decades. One of the main reasons is that the frequencies of the quasinormal oscillations appeared in the dynamical evolution carry the characteristic information about the black hole, which could provide a way for astrophysicists to identify whether there exists black hole in our Universe or not [1 3$]$. The further studies indicate that the quasinormal spectrum could help us to understand more deeply about the quantum gravity [4 6] and the AdS/CFT correspondence [7-9]. Moreover, the stability of a black hole can be examined by the study of the dynamical behaviors of the perturbations in the background spacetime [10-13]. Therefore, a lot of attention have been focused on the dynamical evolution of various perturbations in the various black holes spacetime.

The simplest field in the quantum field theory is scalar field, which associated with spin-0 particles. The dynamical evolution of the scalar field in the different black hole spacetimes has been investigated extensively. It is found that for usual scalar field the late-time evolution after the quasinormal oscillations is dominated by the form $t^{-(2 l+3)}$ for the massless field [14 16] and by the oscillatory inverse power-law form $t^{-(l+3 / 2)} \sin \mu t$ for the massive one [17, 18]. Moreover, the dynamical evolution of the scalar field has also been considered in the cosmology, which shows that the scalar field can be presented as the inflaton to drive the inflation of the

early Universe [19] and as the dark energy to drive the accelerated expansion of the current Universe [20 22]. However, the above investigations are limited to the case where the action has a form

$$
S=\int d^{4} x \sqrt{-g}\left[\frac{R}{16 \pi G}+\frac{1}{2} \partial_{\mu} \psi \partial^{\mu} \psi+V(\psi)+\xi R \psi^{2}\right]+S_{m}
$$

where $\psi, R$ and $V(\psi)$ are corresponding to scalar field, Ricci scalar and scalar potential, respectively. In this action, the coupling between the scalar field and the spacetime curvature contains only the term $\xi R \psi^{2}$, which represents the coupling between the scalar field and the Ricci scalar curvature.

Theoretically, the general form of the action with more couplings between the scalar field and the spacetime curvature can be expressed as

$$
S=\int d^{4} x \sqrt{-g}\left[f\left(\psi, R, R_{\mu \nu} R^{\mu \nu}, R_{\mu \nu \rho \sigma} R^{\mu \nu \rho \sigma}\right)+K\left(\psi, \partial_{\mu} \psi \partial^{\mu} \psi, \nabla^{2} \psi, R^{\mu \nu} \partial_{\mu} \psi \partial_{\nu} \psi, \cdots\right)+V(\psi)\right]+S_{m}
$$

where $f$ and $K$ are arbitrary functions of the corresponding variables. Obviously, the nonlinear functions $f$ and $K$ provide the more non-minimal couplings between the scalar field and the curvature of the background spacetime. These new couplings modify the usual Klein-Gordon equation so that the motion equation for 
the scalar field is no longer generally a second-order differential equation in this case, which yields the more complicated behavior of the scalar field in the background spacetime. By introducing the derivative coupling term $R_{\mu \nu} \partial^{\mu} \psi \partial^{\nu} \psi$, Amendola [23] studied recently the dynamical evolution of the coupled scalar field in the cosmology and obtained some new analytical inflationary solutions. Capozziello et al. [24, 25] investigated a more general model with two coupling terms $R \partial_{\mu} \psi \partial^{\nu} \psi$ and $R_{\mu \nu} \partial^{\mu} \psi \partial^{\nu} \psi$, and found that the de Sitter spacetime is an attractor solution in this case. Recently, Sushkov found [26] that the equation of motion for the scalar field can be reduced to second-order differential equation when it is kinetically coupled to the Einstein tensor. This means that the theory is a "good" dynamical theory from the point of view of physics. Moreover, Sushkov [26] also found that in cosmology the problem of graceful exit from inflation with the derivative coupling term $G^{\mu \nu} \partial_{\mu} \psi \partial_{\nu}$ has a natural solution without any fine-tuned potential. Recently, Gao 27] investigated the cosmic evolution of a scalar field with the kinetic term coupling to more than one Einstein tensors, and found that the scalar field behaves exactly as the pressureless matter if the kinetic term is coupled to one Einstein tensor and acts nearly as a dynamic cosmological constant if it couples with more than one Einstein tensors. The similar investigations have been considered in Refs. [28, 29]. We studied the greybody factor and Hawking radiation for a scalar field coupling to Einstein's tensor in the background of ReissnerNordström black hole spacetime and found that the presence of the coupling enhances both the absorption probability and Hawking radiation of the black hole [30]. These results may excite more efforts to be focused on the study of the scalar field coupled with tensors in the more general cases. The main purpose of this paper is to investigate the dynamical evolution of the scalar perturbation coupling to the Einstein tensor $G^{\mu \nu}$ in the Reissner-Nordström black hole spacetime and see the effect of the coupling on the stability of the black hole.

The plan of our paper is organized as follows: in the following section we will introduce the action of a scalar field coupling to Einstein's tensor and derive its master equation in the Reissner-Nordström black hole spacetime. In Sec.III, we will study the effect of the coupling on the quasinormal modes in the weaker coupling, and then examine the stability of the black hole in the stronger coupling. Finally, in the last section we will include our conclusions.

\section{THE WAVE EQUATION OF A SCALAR FIELD COUPLING TO EINSTEIN'S TENSOR IN THE REISSNER-NORDSTRÖM BLACK HOLE SPACETIME}

In order to study the dynamical evolution of a scalar field coupling to Einstein's tensor in a black hole spacetime, we must first obtain its wave equation in the background. The action of the scalar field coupling 
to the Einstein's tensor $G^{\mu \nu}$ in the curved spacetime has a form [26],

$$
S=\int d^{4} x \sqrt{-g}\left[\frac{R}{16 \pi G}+\frac{1}{2} \partial_{\mu} \psi \partial^{\mu} \psi+\frac{\eta}{2} G^{\mu \nu} \partial_{\mu} \psi \partial_{\nu} \psi\right]
$$

The coupling between Einstein's tensor $G^{\mu \nu}$ and the scalar field $\psi$ is represented by the term $\frac{\eta}{2} G^{\mu \nu} \partial_{\mu} \psi \partial_{\nu} \psi$, where $\eta$ is coupling constant with dimensions of length-squared.

Varying the action (3) with respect to $\psi$, one can find the wave equation of a scalar field coupling to Einstein's tensor can be expressed as [26, 30]

$$
\frac{1}{\sqrt{-g}} \partial_{\mu}\left[\sqrt{-g}\left(g^{\mu \nu}+\eta G^{\mu \nu}\right) \partial_{\nu} \psi\right]=0 .
$$

Obviously, the dynamical evolution of a scalar field depends on the the Einstein's tensor $G^{\mu \nu}$ and the coupling constant $\eta$. Since all the components of the tensor $G^{\mu \nu}$ vanish in the Schwarzschild black hole spacetime, we cannot probe the effect of the coupling term on the dynamical behavior of the scalar perturbation. In the general relative theory, the simplest black hole with the non-zero components of the tensor $G^{\mu \nu}$ is ReissnerNordström one, whose metric has a form

$$
d s^{2}=-f d t^{2}+\frac{1}{f} d r^{2}+r^{2} d \theta^{2}+r^{2} \sin ^{2} \theta d \phi^{2}
$$

with

$$
f=1-\frac{2 M}{r}+\frac{q^{2}}{r^{2}}
$$

where $M$ is the mass and $q$ is the charge of the black hole. The Einstein's tensor $G^{\mu \nu}$ for the metric (5) has a form

$$
G^{\mu \nu}=\frac{q^{2}}{r^{4}}\left(\begin{array}{cccc}
-\frac{1}{f} & & & \\
& f & & \\
& & -\frac{1}{r^{2}} & \\
& & -\frac{1}{r^{2} \sin ^{2} \theta}
\end{array}\right) .
$$

Defining tortoise coordinate $d r_{*}=1 / f(r) d r$ and separating $\psi(t, r, \theta, \phi)=\frac{e^{-i \omega t} R(r) Y_{l m}(\theta, \phi) r}{\sqrt{r^{4}+\eta q^{2}}}$, we can obtain the radial equation for the scalar perturbation coupling to Einstein's tensor in the Reissner-Nordström black hole spacetime

$$
\frac{d^{2} R(r)}{d r_{*}^{2}}+\left[\omega^{2}-V(r)\right] R(r)=0
$$

with the effective potential

$$
V(r)=f\left(\frac{r^{4}-\eta q^{2}}{r^{4}+\eta q^{2}}\right)\left[\frac{l(l+1)}{r^{2}}+\frac{f^{\prime}}{r}\right]+\frac{f^{2}}{r^{2}} \frac{2 \eta q^{2}\left(3 r^{4}+\eta q^{2}\right)}{\left(r^{4}+\eta q^{2}\right)^{2}} .
$$


Obviously, as the coupling constant $\eta=0$ the radial equation (8) reduces to that of the scalar one without coupling to Einstein's tensor. In the case $\eta \neq 0$, the coupling constant $\eta$ emerges in the effective potential, which means that coupling between the scalar perturbation and Einstein's tensor will change the dynamical evolution of the scalar perturbation in the background spacetime. In Fig.1, we plot the changes of the
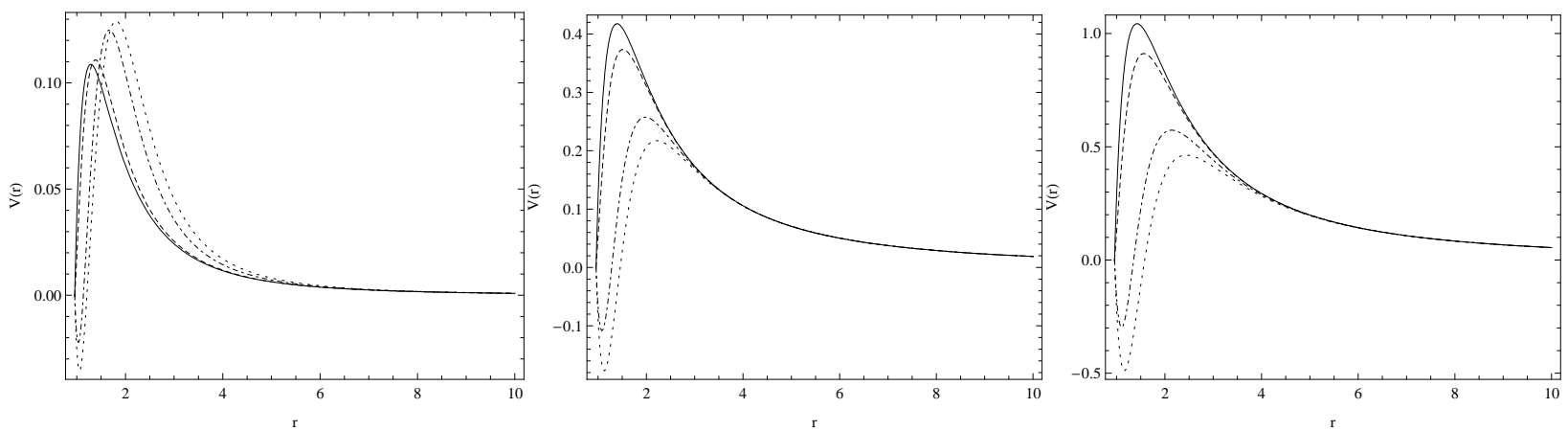

FIG. 1: Variety of the effective potential $V(r)$ with the polar coordinate $r$ for fixed $l=0$ (left), $l=1$ (middle) and $l=2$ (right). The solid, dashed, dash-dotted and dotted lines are corresponding to the cases with $\eta=0,10,100,200$, respectively. We set $2 M=1$ and $q=0.2$.

effective potential $V(r)$ with the coupling constant $\eta$ for fixed $l$ and $q$. With increase of $\eta$, the peak height of the potential barrier increases for $l=0$ and decreases for other values of $l$. Moreover, one can find that for the smaller $\eta$ the effective potential $V(r)$ is positive definite everywhere outside the black hole event horizon. This implies that the solution of the wave equation (4) is bounded and the black hole is stable in this case. However, for the larger $\eta$, we find that the effective potential $V(r)$ has negative gap, and then the stability is not guaranteed. In the following section, we will check those values of $\eta$ for which the negative gap is present and study the stability of the black hole when the scalar perturbation is coupling to Einstein's tensor.

\section{THE INSTABILITY OF SCALAR FIELD COUPLING TO EINSTEIN'S TENSOR IN THE BACKGROUND OF A REISSNER-NORDSTRÖM BLACK HOLE}

In this section, we first consider the quasinormal modes in the weaker coupling case in which the effective potential $V(r)$ is positive definite and study the effects of the coupling on the quasinormal frequencies. Then, we shall study the evolution of the scalar field coupling to Einstein's tensor in time domain using a numerical characteristic integration method [31] and check the instability of the black hole in the stronger coupling.

Let us now to study the effects of the coupling constant on the massless scalar quasinormal modes in the Reissner-Nordström black hole spacetime in the weaker coupling case. In Fig.2 and 3, we present the fundamental quasinormal modes $(n=0)$ evaluated by the third-order WKB approximation method [32, 33 . It is shown that with the increase of the coupling constant $\eta$ the real parts of the quasinormal frequencies 
decrease and the absolute values of imaginary parts increases for fixed $l$ and $q$. This means that the presence
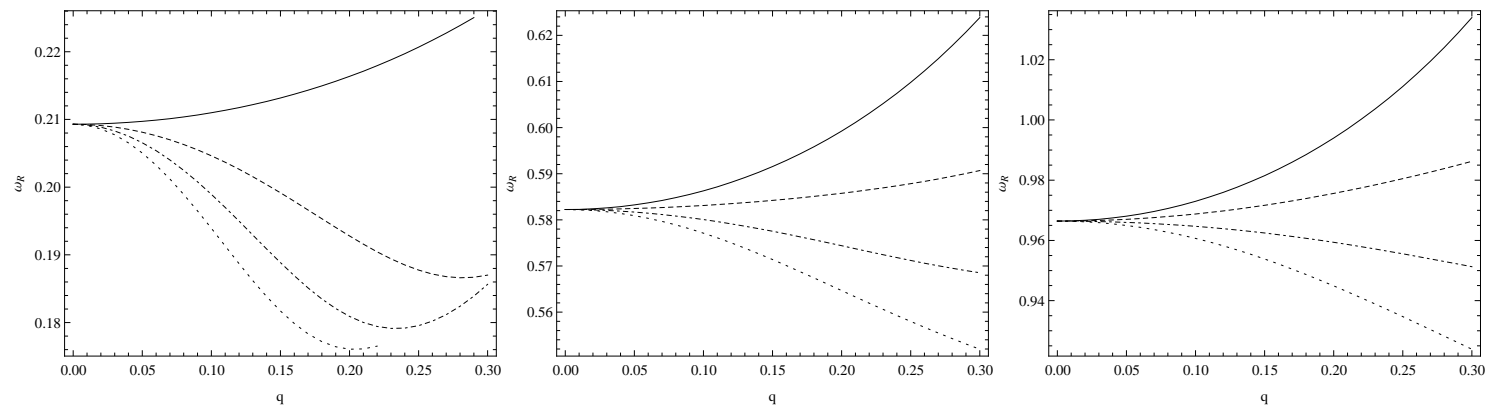

FIG. 2: Variety of the real parts of the fundamental quasinormal modes with $q$ for scalar field coupling to Einstein's tensor in the Reissner-Nordström black hole spacetime. The figures from left to right are corresponding to $l=0,1$ and 2. The solid, dashed, dash-dotted and dotted lines are corresponding to the cases with $\eta=0,2,4,6$, respectively. We set $2 M=1$.
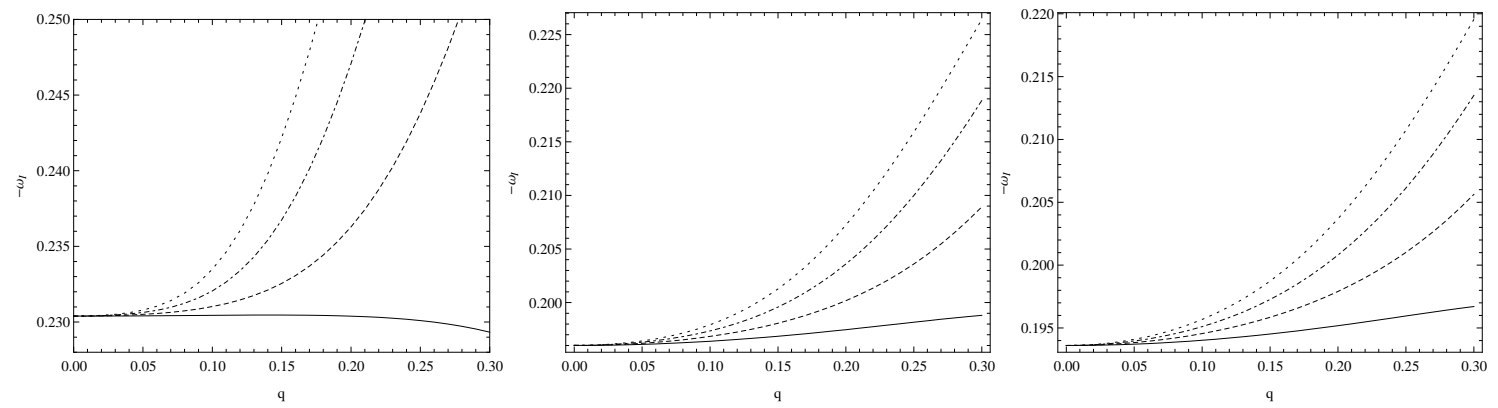

FIG. 3: Variety of the absolute value of imaginary parts of the fundamental quasinormal modes with $q$ for scalar field coupling to Einstein's tensor in the Reissner-Nordström black hole spacetime. The figures from left to right are corresponding to $l=0,1$ and 2. The solid, dashed, dash-dotted and dotted lines are corresponding to the cases with $\eta=0,2,4,6$, respectively. We set $2 M=1$.

of the coupling parameter $\eta$ makes the decay of the scalar perturbation more quickly in this case. From Fig.2 and 3 , one can easily obtain that with increase of $q$ the real parts $\omega_{R}$ increases for the smaller $\eta$ and decreases for the larger $\eta$. The changes of the absolute values of imaginary parts with $q$ are more complicated. For $l=0$, it decreases for the smaller $\eta$ and increases for the larger $\eta$, while for other values of $l$, it increase with $q$ for all $\eta$. These results imply that the presence of the coupling terms modifies the standard results in the quasinormal modes of the scalar perturbations in the background of a Reissner-Nordström black hole.

We are now in a position to study the dynamical evolution of the scalar field coupling to Einstein's tensor in time domain and examine the stability of the black hole in the stronger coupling cases. Adopting to the light-cone variables $u=t-r_{*}$ and $v=t+r_{*}$, one can find that the wave equation

$$
-\frac{\partial^{2} \psi}{\partial t^{2}}+\frac{\partial^{2} \psi}{\partial r_{*}^{2}}=V(r) \psi,
$$


can be rewritten as

$$
4 \frac{\partial^{2} \psi}{\partial u \partial v}+V(r) \psi=0
$$

This two-dimensional wave equation (11) can be integrated numerically by using the finite difference method
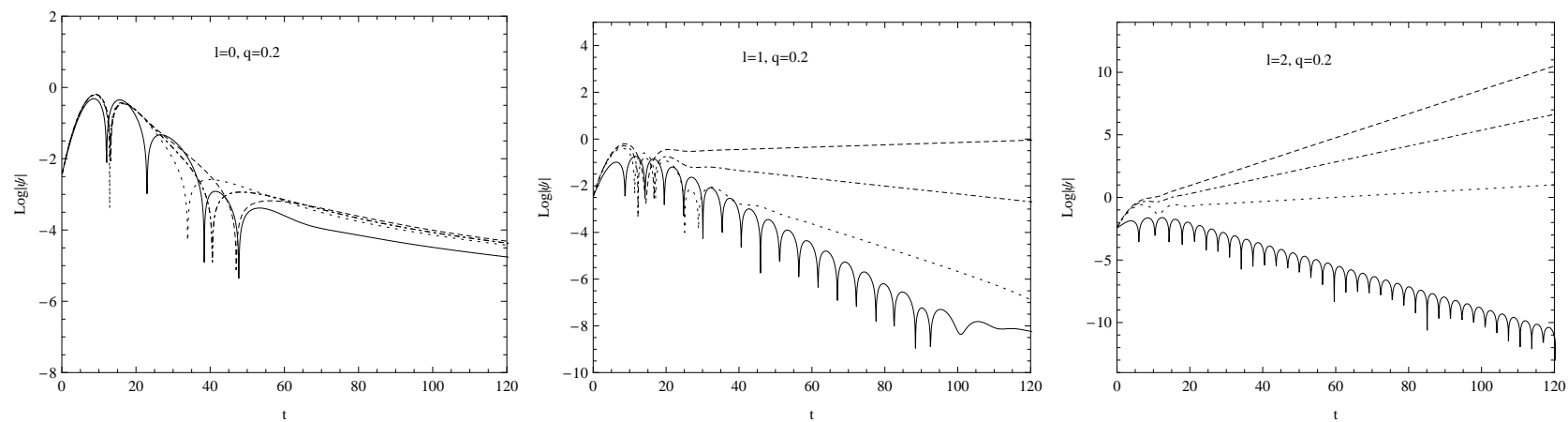

FIG. 4: The dynamical evolution of a scalar field coupling to Einstein's tensor in the background of a ReissnerNordström black hole spacetime. The figures from left to right are corresponding to $l=0,1$ and 2 . The solid, dashed, dash-dotted and dotted lines are corresponding to the cases with $\eta=1,60,80,100$, respectively. We set $2 M=1$. The constants in the Gauss pulse (13) $v_{c}=10$ and $\sigma=3$.

suggested in [31]. In terms of Taylor's theorem, it can be discretized as

$$
\psi_{N}=\psi_{E}+\psi_{W}-\psi_{S}-\delta u \delta v V\left(\frac{v_{N}+v_{W}-u_{N}-u_{E}}{4}\right) \frac{\psi_{W}+\psi_{E}}{8}+O\left(\epsilon^{4}\right)=0,
$$

where we have used the following definitions for the points: $N:(u+\delta u, v+\delta v), W:(u+\delta u, v), E:(u, v+\delta v)$ and $S:(u, v)$. The parameter $\epsilon$ is an overall grid scalar factor, so that $\delta u \sim \delta v \sim \epsilon$. Since the behavior of the wave function is not sensitive to the choice of initial data, we can set $\psi\left(u, v=v_{0}\right)=0$ and use a Gaussian pulse as an initial perturbation, centered on $v_{c}$ and with width $\sigma$ on $u=u_{0}$ as

$$
\psi\left(u=u_{0}, v\right)=e^{-\frac{\left(v-v_{c}\right)^{2}}{2 \sigma^{2}}}
$$

In fig.4, we present the dynamical evolution of the scalar field coupling to Einstein's tensor in the background of a Reissner-Nordström black hole. For the coupling constant $\eta=1$, the decay of the coupling scalar field is similar to that of the scalar one without coupling to Einstein's tensor, which indicates that the black hole is stable in the weaker coupling. It is expectable because the effective potential $V(r)$ is positive definite in this cases. For $l=0$, we also note that the scalar field always decays for any value of the coupling constant $\eta$. This means that the lowest $l$ are stable, which can be explained by a fact that for $l=0$, the higher $\eta$ raise up the peak of the potential barrier so that the potential is always positive definite. Moreover, for the higher multipole numbers $l$, we find that the scalar field grows with exponential rate as the coupling constant $\eta$ is 


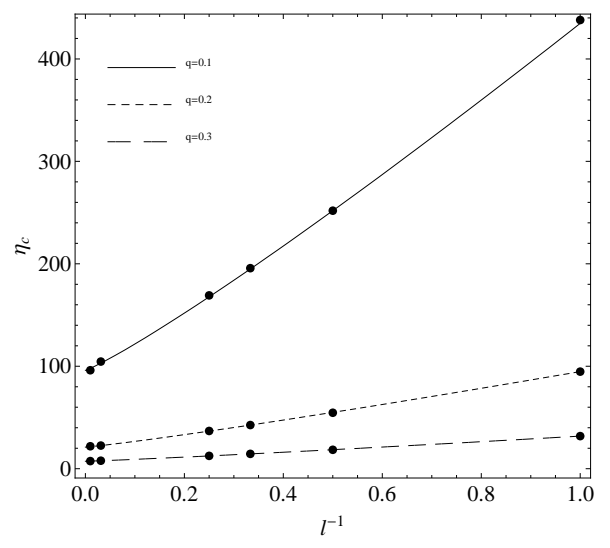

FIG. 5: The change of the threshold value $\eta_{c}$ with the inverse multipole number $l^{-1}$ for fixed $q$. The points $l=$ $1,2,3,4,32,100$ were fitted by the function $\eta_{c}=a l^{-1.12}+b$. The values of $(a, b)$ for $q=0.1,0.2$ and 0.3 are $(338.71,96.00),(73.72,21.10)$ and $(24.52,7.31)$, respectively.

larger than the critical value $\eta_{c}$, which means that the instability occurs in this case. The main reason is that for $l \neq 0$ the large $\eta$ drops down the peak of the potential barrier and increases the negative gap near the black hole horizon so that the potential could be non-positive definite. In the instability region, the larger $\eta$, the instability growth occurs at the earlier times, and the growth rate is the stronger. Furthermore, we plotted the change of the threshold value $\eta_{c}$ with $l$ in fig.5, and found that the threshold value can be fitted best by the function

$$
\eta_{c} \simeq a l^{-1.12}+b
$$

where $a$ and $b$ are numerical constants. It is easy to obtain that the lowest $l$ are stable because the threshold
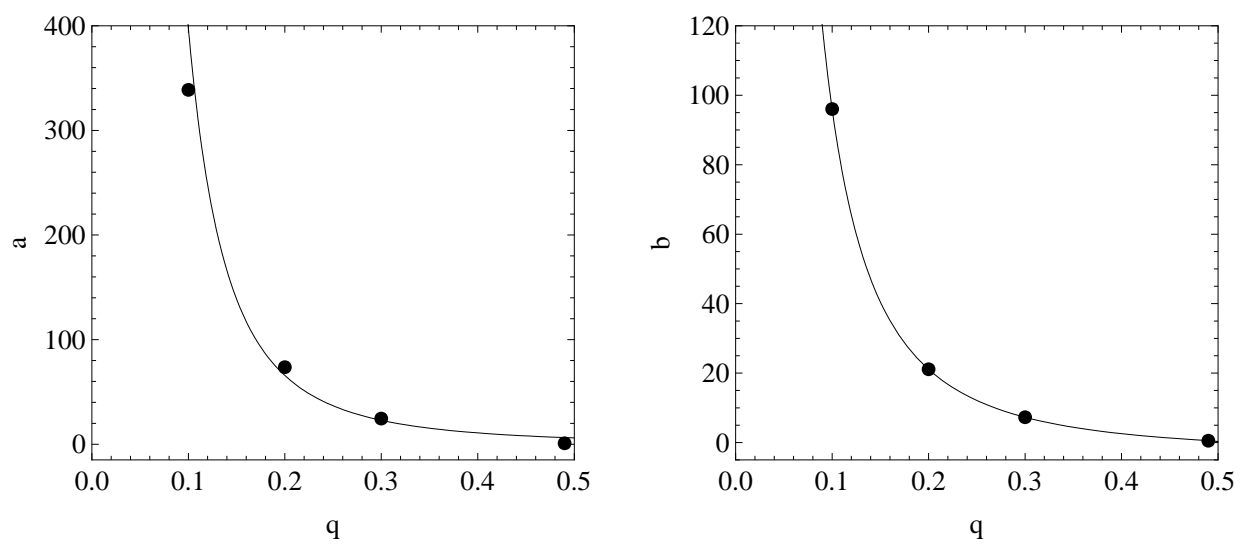

FIG. 6: The changes of the numerical constants $a, b$ with the charge $q$, which were fitted by the functions $a=q^{-2.6}$ and $b=r_{+}^{4} / q^{2}$, respectively.

value $\eta_{c} \rightarrow \infty$ as $l \rightarrow 0$. Moreover, for the higher $l$ and smaller $q$, we have the smaller threshold value $\eta_{c}$ at which instability happens. The varieties of the numerical constants $a, b$ with $q$ are presented in fig.6, which 
shows that the values of $a, b$ are fitted best by the functions $a \simeq q^{-2.6}$ and $b \simeq r_{+}^{4} / q^{2}$, respectively. Thus, as the charge $q$ vanishes, the threshold value $\eta_{c}$ tends to infinite for arbitrary $l$, which means that the Schwarzschild black hole is stable when it is perturbed by a scalar field coupling to Einstein's tensor. Actually, since all the components of the Einstein's tensor disappear in the Schwarzschild black hole spacetime, the dynamical evolution of the coupling scalar field is consistent with that of the scalar one without coupling to Einstein's tensor. For the extreme black hole, we find from fig.6 and Eq.(14) that the threshold value $\eta_{c}$ is the minimum for arbitrary $l \neq 0$, which implies that the instability happens more easily in the extreme black hole. From the previous discussions, we can obtain that in the limit $l \rightarrow \infty$ the threshold value $\eta_{c} \rightarrow b$ and the effective potential (9) has the form

$$
\left.V(r)\right|_{l \rightarrow \infty}=f\left(\frac{r^{4}-\eta q^{2}}{r^{4}+\eta q^{2}}\right) \frac{l(l+1)}{r^{2}} .
$$

According to the method suggested in [34], the integration

$$
\int_{r_{+}}^{\infty} \frac{\left.V(r)\right|_{l \rightarrow \infty}}{f} d r=\int_{r_{+}}^{\infty}\left(\frac{r^{4}-\eta q^{2}}{r^{4}+\eta q^{2}}\right) \frac{l(l+1)}{r^{2}} d r
$$

is positive definite as $\eta<r_{+}^{4} / q^{2}$. It implies that the threshold value has a form $\eta_{c}=r_{+}^{4} / q^{2}$ as $l \rightarrow \infty$, which is consistent with the form of the numerical constant $b$ obtained in the previous numerical calculation.

\section{SUMMARY}

In this paper, we have studied the dynamical evolution of a scalar field coupling to Einstein's tensor in the background of Reissner-Nordström black hole. Our results show that the the coupling constant $\eta$ imprints in the wave dynamics of a scalar perturbation. For the multipole number $l=0$, we find that the scalar field always decays for arbitrary coupling constant $\eta$. For $l \neq 0$, the instability occurs when $\eta$ is larger than the critical value $\eta_{c}$. Moreover, for the higher $l$, we have the smaller threshold value $\eta_{c}$. In the weak coupling (i.e., $\eta \ll \eta_{c}$ ) case, we find that with the increase of the coupling constant $\eta$ the real part of the fundamental quasinormal frequencies decreases and the absolute value of imaginary parts increases for fixed $l$ and $q$. With increase of $q$ the real part $\omega_{R}$ increases for the smaller $\eta$ and decreases for the larger $\eta$. For $l=0$, the absolute value of imaginary parts decreases for the smaller $\eta$ and increases for the larger $\eta$, while for other values of $l$, it increases with $q$ for all $\eta$. Moreover, we find that the threshold value can be fitted best by the function $\eta_{c} \simeq a l^{-1.12}+b$ and the numerical constants $a, b$ decrease with the charge $q$. These rich dynamical properties of the scalar field coupling to Einstein's tensor, which could provide a way to detect whether there exist a coupling between the scalar field and Einstein's tensor or not. It would be of interest to generalize our study 
to other black hole spacetimes, such as rotating black holes etc. Work in this direction will be reported in the future.

\section{ACKNOWLEDGMENTS}

This work was partially supported by the National Natural Science Foundation of China under Grant No.10875041, the Program for Changjiang Scholars and Innovative Research Team in University (PCSIRT, No. IRT0964) and the construct program of key disciplines in Hunan Province. J. Jing's work was partially supported by the National Natural Science Foundation of China under Grant Nos. 10875040 and 10935013 ; 973 Program Grant No. 2010CB833004 and the Hunan Provincial Natural Science Foundation of China under Grant No.08JJ3010.

[1] S. Chandrasekhar and S. Detweller, Proc. R. Soc. Lond. A 344, 441 (1975).

[2] H. P. Nollert, Class. Quantum Grav. 16, R159 (1999).

[3] K. D. Kokkotas and B. G. Schmidt, Living Rev. Rel. 2, 2 (1999).

[4] S. Hod, Phys. Rev. Lett. 81, 4293 (1998).

[5] O. Dreyer, Phys. Rev. Lett. 90, 081301 (2003).

[6] A. Corichi, Phys. Rev. D 67, 087502 (2003); L. Motl, Adv. Theor. Math. Phys. 6, 1135 (2003); L. Motl and A. Neitzke, Adv. Theor. Math. Phys. 7, 307 (2003); A. Maassen van den Brink, J. Math. Phys. 45, 327 (2004); G. Kunstatter, Phys. Rev. Lett. 90, 161301 (2003); N. Andersson and C. J. Howls, Class. Quantum Grav. 21, 1623 (2004); V. Cardoso, J. Natario and R. Schiappa, J. Math. Phys. 45, 4698 (2004); J. Natario and R. Schiappa, Adv. Theor. Math. Phys. 8, 1001 (2004); V. Cardoso and J. P. S. Lemos, Phys. Rev. D 67, 084020 (2003).

[7] J. Maldacena, Adv. Theor. Math. Phys. 2, 231 (1998).

[8] E. Witten, Adv. Theor. Math. Phys. 2, 253 (1998).

[9] G. T. Horowitz and V. E. Hubeny, Phys. Rev. D 62, 024027 (2000); B. Wang, C. Y. Lin and E. Abdalla, Phys. Lett. B 481, 79 (2000) ; J. M. Zhu, B. Wang and E. Abdalla, Phys. Rev. D 63, 124004 (2001); V. Cardoso and J. P. S. Lemos, Phys. Rev. D 63, 124015 (2001); V. Cardoso and J. P. S. Lemos, Phys. Rev. D 64, 084017 (2001); E. Berti and K. D. Kokkotas, Phys. Rev. D 67, 064020 (2003); E. Winstanley, Phys. Rev. D 64, 104010 (2001); J. S. F. Chan and R. B. Mann, Phys. Rev. D 59, 064025 (1999).

[10] R. Gregory and R. Laflamme, Phys. Rev. Lett. 70, 2837 (1993); R. Gregory and R. Laflamme, Nucl. Phys. B 428, 399 (1994).

[11] T. Harmark, V. Niarchos and N. A. Obers, Class. Quant. Grav. 24, R1 (2007).

[12] R. A. Konoplya, K. Murata, Jiro Soda and A. Zhidenko, Phys. Rev. D 78, 084012 (2008); J. L. Hovdebo and R. C. Myers, Phys. Rev. D 73, 084013 (2006).

[13] S. B. Chen and J. L. Jing, JHEP 03, 081 (2009).

[14] R. H. Price, Phys. Rev. D 5, 2419 (1972). 
[15] S. Hod and T. Piran, Phys. Rev. D 58, 024017 (1998).

[16] L. Barack, Phys. Rev. D 61, 024026 (2000); L. M. Burko and G. Khanna, Phys. Rev. D 67, 081502 (2003); E. S. C. Ching, P. T. Leung, W. M. Suen and K. Young, Phys. Rev. D 52, 2118 (1995).

[17] H. Koyama and A. Tomimatsu, Phys. Rev. D 63, 064032 (2001); H. Koyama and A. Tomimatsu, Phys. Rev. D 64, 044014 (2001); R. Moderski and M. Rogatko, Phys. Rev. D 64, 044024 (2001); R. Moderski and M. Rogatko, Phys. Rev. D 63, 084014 (2001); R. Moderski and M. Rogatko, Phys. Rev. D 72, 044027 (2005); S. Hod and T. Piran, Phys. Rev. D 58, 044018 (1998). S. B. Chen and J. L. Jing, Mod. Phys. Lett. A 23, 35 (2008); S. B. Chen, B. Wang and R. K. Su, Int. J. Mod. Phys. A 16, 2502 (2008).

[18] S. Hod, Phys. Rev. D 58, 104022 (1998) ; L. Barack and A. Ori, Phys. Rev. Lett. 82, 4388 (1999) ; W. krivan, Phys. Rev. D 60, 101501(R) (1999); Q. Y. Pan and J. L. Jing, Chin. Phys. Lett. 21, 1873 (2004).

[19] A. H. Guth, Phys. Rev. D 23, 347 (1981). 1a

[20] B. Ratra and J. Peebles, Phys. Rev. D 37, 3406 (1988); C. Wetterich, Nucl. Phys. B 302, 668 (1988); R. R. Caldwell, R. Dave and P. J. Steinhardt, Phys. Rev. Lett. 80, 1582 (1988) ; M. Doran and J. Jaeckel, Phys. Rev. D 66, 043519 (2002).

[21] C. A. Picon, T. Damour and V. Mukhanov, Phys. Lett. B 458, 209 (1999); T. Chiba, T. Okabe and M. Yamaguchi, Phys. Rev. D 62, 023511 (2000).

[22] R. R. Caldwell, Phys. Lett. B 545, 23 (2002) ; B. McInnes, J. High Energy Phys. 08, 029 (2002); S. Nojiri and S. D. Odintsov, Phys. Lett. B 562, 147 (2003); L. P. Chimento and R. Lazkoz, Phys. Rev. Lett. 91, 211301 (2003); B. Boisseau, G. Esposito-Farese, D. Polarski, Alexei A. Starobinsky, Phys. Rev. Lett. 85, 2236 (2000); R. Gannouji, D. Polarski, A. Ranquet, A. A. Starobinsky, JCAP 0609, 016 (2006).

[23] L. Amendola, Phys. Lett. B 301, 175 (1993).

[24] S. Capozziello, G. Lambiase and H. J.Schmidt, Annalen Phys. 9, 39 (2000).

[25] S. Capozziello, G. Lambiase, Gen. Rel. Grav. 31, 1005 (1999) .

[26] S. V. Sushkov, Phys. Rev. D 80, 103505 (2009).

[27] C. J. Gao, JCAP 06, 023 (2010), arXiv: 1002.4035.

[28] L.N. Granda, arXiv: 0911.3702.

[29] E. N. Saridakis and S. V. Sushkov, Phys. Rev. D 81, 083510 (2010), arXiv: 1002.3478.

[30] S. B. Chen and J. L. Jing, arXiv: 1005.5601.

[31] C. Gundlach, R. H. Price and J. Pullin, Phys. Rev. D 49, 883 (1994).

[32] B. F. Schutz and C. M. Will, Astrophys. J. Lett. 291, L33 (1985).

[33] S. Iyer and C. M. Will, Phys. Rev. D 35, 3621 (1987); S. Iyer, Phys. Rev. D 35, 3632 (1987).

[34] R. J. Gleiser and G. Dotti, Phys. Rev. D 72, 124002 (2005); W. F. Buell and B. A. Shadwick, Am. J. Phys. 63, 256 (1995). 